Post-print version

Copyright: American Society of Civil Engineers

Source: http://ascelibrary.org/doi/abs/10.1061/(ASCE)WR.1943-5452.0000535

\title{
Sensitivity Analysis for Sampling Design and Demand Calibration in Water Distribution Networks using the Singular Value Decomposition
}

\author{
Gerard Sanz ${ }^{1}$ and Ramon Pérez ${ }^{2}$
}

\begin{abstract}
Research in water distribution networks during last decades has often focused on calibration. There is no unique solution for this problem as the methodologies are developed depending on which parameters have to be calibrated and the final use of the model. This work presents a demand calibration methodology that identifies a set of patterns that minimise the error in predicted measurements. The singular value decomposition (SVD) of the sensitivity matrix is a powerful tool for solving the optimisation problem. Additionally, in this work, the deep understanding of the SVD allows the selection of an alternative to the classic patterns. Each individual demand is defined as a combination of geographically distributed patterns. The membership of each demand to every pattern is produced naturally through the analysis of the SVD of the sensitivity matrix. Three types of memberships are considered: binary, positive and free. The SVD analysis is also used to define the location of sensors for the calibration. The performance of the methodology proposed is tested on a real water distribution network using synthetic data. Results show that the use of positive memberships to define individual demands is the best option to reduce the error in predicted pressures and flows.
\end{abstract}

Keywords: Calibration, Demands, Sampling Design, Patterns, Water Distribution Systems

\section{INTRODUCTION}

Water Distribution Network (WDN) models are used by water companies in a wide range of applications. A good calibration of these models is required in order to improve the confidence of the application results (Sumer and Lansey 2009). Walski (1983) assessed that the major uncertainties in water distribution models

\footnotetext{
${ }^{1} \mathrm{PhD}$ student in Dept. of Automatic Control, Polytechnic University of Catalonia, Terrassa 08222. E-mail: gerard.sanz@upc.edu

${ }^{2}$ Associate Professor in Dept. of Automatic Control, Polytechnic University of Catalonia, Terrassa 08222. E-mail: ramon.perez@upc.edu
} 
are pipes roughness and nodal consumptions. More recently, Goulet et al. (2013) pointed out that the most important uncertainty sources are demands and model simplifications. The authors' experience in WDN monitoring and the operators' feedback are in agreement that demands are the most uncertain parameters. This was confirmed by the study of the effect of a bad calibration on leak localisation (Pérez et al. 2011). The continuous evolution of demands also justifies the realtime calibration to keep the model updated, whereas roughness is a more stable parameter that can be estimated offline from manufacturer information.

Water networks are generally composed of thousands of pipes and nodes. However, the number of measurements taken is reduced to a few selected locations. Consequently, it is unfeasible to calibrate thousands of individual demands. The identifiability of a system is the capacity for estimating its parameters using measurements. In order to improve the identifiability new measurements have to be introduced or the number of parameters has to be reduced.

The aim of this work is to identify a set of demand patterns that minimise the error in predicted measurements. These patterns are calibrated continuously in time, and have a spatial distribution obtained from a pressure sensitivity analysis. Nodal demands are then generated as a combination of these patterns weighted by their base demand, which is obtained from billing.

The high connectivity between elements in WDN has to be considered when placing sensors in order to avoid redundant measurements. This paper proposes a sampling design methodology that maximises the information obtained from the network measurements for the calibration process.

Finally, calibration as an inverse problem is formulated and solved. Demand patterns are estimated online in an iterative process that minimises the errors in predicted measurements.

The Singular Value Decomposition (SVD) and its properties are used to analyse the sensitivity matrix of the WDN for solving the three mentioned steps: parameterization, sampling design and calibration. The validity of the proposed methods is evaluated using a real network with synthetic data.

These methodologies require the availability of the hydraulic model of the network. The boundary conditions of the network have to be known to simulate the model and to obtain the predicted measures. These predictions will be compared with the measurements from the installed sensors placed by the sampling design methodology. Historical data from quarterly billing is also required as "a priori" information to enhance the calibration results.

A note about notation: Scalars will be referred to by lower case letters; vectors (always considered as column matrices) will be referred to by lower case boldface letters; and matrices will be referred to by upper case boldface letters. A complete list of symbols is provided at the end of the paper, after the conclusions section.

\section{REVIEW OF PREVIOUS RESEARCH}

The calibration problem in WDN is a great challenge due to the presence of high uncertainty together with the availability of few measurements. Shamir and Howard (1977) stated that calibration "consists of determining the physical and 
operational characteristics of an existing system and determining the data [that] when input to the computer model will yield realistic results".

Savic et al. (2009) presented a complete review of the global calibration state of the art in WDN. Methods were classified depending on the model dynamics (static/transient) and depending on the optimisation technique: (1) Iterative methods, based on trial and error procedures; (2) Explicit methods, which solve the extended set of steady-state mass balance and energy equations; and (3) Implicit methods, which are formulated and solved using an optimisation technique coupled with a hydraulic solver.

This work proposes an implicit method for calibrating demands. The optimisation problem can be solved using different approaches. The most used ones in literature are weighted-least-squares methods based on Gauss-Newton (Kang and Lansey 2011), Genetic Algorithms and other global optimisation methods (Nicolini et al. 2011). The SVD was used by Wiggins (1972) and Uhrhammer (1980) for seismographic networks, by Wasantha Lal (1995) for unsteady river flow networks and by Cheng and He (2011) for WDN. The SVD provides a deep comprehension of the calibration problem, encouraging its adaption in the current study to estimate demand patterns in WDN.

Due to the inaccuracy of the input data, it is important to determine the reliability of the estimations. Most of the previous research reviewed performed the quantification of the parameter and prediction uncertainties by means of the FirstOrder Second-Moment model (FOSM) based on linear regression theory. Hutton et al. (2014) reviewed some promising approaches for uncertainty quantification and reduction in the modelling cascade.

The methodology presented in this work focuses on the calibration of demands. Any place where water can leave the system represents a point of consumption, including a customer's faucet, a leaky main, or an open fire hydrant. These water consumptions are the driving force behind the hydraulic dynamics occurring in WDN (Walski et al. 2003).

Water usage in municipal WDN is inherently unsteady due to continuously varying demands. To be effective, a real-time modelling technique must accommodate the disconnection between mean demand estimates that change gradually and real demands. The objectives of this work, as stated in the introduction, are the time and spatial distribution of demands (Davidson and Bouchart 2006). Therefore, the calibration of demands proposed will use both information coming from outside (billing records) and inside (installed sensors) the network.

\section{METHODOLOGY}

This section presents a detailed description of the developed methodology. First, the mathematical framework of the generalised inverse problem and the SVD solution are described. Next, the problem is detailed for demand calibration in WDN. Then, the methodology for selecting a new set of parameters that represent the demand behaviour is presented. Finally, the method for selecting the sensors' locations that enhance the calibration results is described. 


\section{The inverse problem}

The objective of the calibration problem is to find the parameter vector $\mathbf{x}$ that minimises the errors $\mathbf{e}=\mathbf{y}_{\mathbf{m}}-\mathbf{y}_{\mathbf{p}}$, where $\mathbf{y}_{\mathbf{m}}$ and $\mathbf{y}_{\mathbf{p}}$ are the vectors of measured and predicted values, respectively. The corrections in parameters $\Delta \mathrm{x}$ that make $\mathbf{e} \rightarrow 0$ are obtained by solving the system of equations:

$$
\mathrm{S} \cdot \Delta \mathrm{x}=\mathrm{y}_{\mathbf{m}}-\mathrm{y}_{\mathbf{p}}
$$

where $\mathbf{S}$ is the sensitivity matrix that relates errors in predictions to corrections in the models' parameters. In non-linear problems, $\Delta \mathbf{x}$ is calculated iteratively and used to correct the parameter vector $\mathbf{x}$ :

$$
\mathbf{x}_{r+1}=\mathbf{x}_{r}+\rho \Delta \mathbf{x}_{r}
$$

where $r$ is the iteration number and $\rho$ is a parameter to control the step size. The iterative scheme is continued until a termination criterion is achieved (Bard 1974).

\section{Singular Value Decomposition}

The system in Eq. 1 can be solved using methods such as Gaussian elimination (evendetermined), least squares method (overdetermined) or Penrose inverse solution (underdetermined). However, none of these solution techniques can be used with rank-deficient or ill-conditioned matrices (Aster et al. 2005). The SVD is capable of solving under-, over-, even- or mixed-determined problems with no rank conditions in $\mathbf{S}$, as explained by Menke (1982). The SVD of matrix $\mathbf{S}$ in Eq. 1 is:

$$
\mathbf{S}=\mathbf{U} \cdot \boldsymbol{\Lambda} \cdot \mathbf{V}^{T}
$$

where $\mathbf{U}$ is a $\mathrm{m} \times \mathrm{m}$ matrix of orthonormal singular vectors associated with the $\mathrm{m}$ observed data, $\mathbf{V}$ is a $\mathbf{n} \times \mathbf{n}$ matrix of orthonormal singular vectors associated with the $\mathrm{n}$ system parameters; and $\boldsymbol{\Lambda}$ is a $\mathrm{m} \times \mathrm{n}$ diagonal matrix of singular values of $\mathbf{S}$, where the additional rows (more measurements than parameters) or columns (more parameters than measurements) are filled with zeros. Eq. 1 can be solved by the SVD as:

$$
\Delta \mathbf{x}=\mathbf{V} \frac{1}{\Lambda} \mathbf{U}^{T} \mathbf{e}
$$

where $1 / \lambda_{i}$ is the ith diagonal element of $1 / \boldsymbol{\Lambda}$, and $\lambda_{i}$ is the ith diagonal element of $\Lambda$ (for $\lambda_{i}=0$, the corresponding element of $1 / \boldsymbol{\Lambda}$ is set to 0 ). The SVD determines the optimisation direction $\Delta \mathrm{x}$ for a problem that minimises $\|\Delta \mathrm{x}\|_{2}$ and $\|\mathbf{e}\|_{2}$. A cut-off level for small $\lambda_{i}$ is set to avoid $1 / \lambda_{i}$ becoming too large. In this work, the cut-off level is defined at a value of $\lambda=10^{-3}$ as suggested by Wiggins (1972) and Wasantha Lal (1995).

Uhrhammer (1980) and Wiggins (1972) used the SVD matrices for the estimation of the parameter space covariance matrix to quantify the uncertainty of the calibrated model (Eq. 5). This estimation corresponds to the FOSM model (Kapelan et al. 2005), but can be applied to both singular and non-singular matrices.

$$
\mathbf{\Upsilon}^{2}=\mathbf{V} \frac{\sigma^{2}}{\Lambda^{2}} \mathbf{V}^{T}
$$


where $\sigma^{2}$ is the variance of the measurements, considered the same for all sensors. The diagonal elements of $\Upsilon$ are estimates of the uncertainty (standard error) of the calibrated parameters.

\section{Sensitivity matrix computation}

The application of the generalised inverse problem for WDN calibration requires the calculation of the sensitivity coefficients, i.e. the partial derivatives of head and flow with respect to each of the calibrated parameters.

Yeh (1986) reviewed three methodologies for the calculation of the sensitivity coefficients in groundwater hydrology: (a) Influence coefficient method; (b) Sensitivity equation method; and (c) Variational method. All three methodologies require $n+1$ simulations to be run in order to compute the complete sensitivity matrix, where $n$ is the number of parameters in the model. The use of these methods for the calculation of the sensitivity matrix in real water networks is unfeasible when calibrating nodal demands.

Cheng and He (2011) proposed a matrix analysis of the WDN linearised model in order to obtain the sensitivity matrix. In this methodology only one simulation is required at each iteration. The matrix model (Bryds and Ulanicki 1994) of the WDN is defined in Eq. 6.

$$
-\mathrm{BCB}^{T} \mathbf{h}=\mathbf{d}
$$

where $\mathbf{B}$ is the incidence matrix of the network; $\mathbf{C}$ is the non-linear matrix depending on the pipes roughness, lengths, diameters and hydraulic gradient; $\mathbf{h}$ is the vector of heads; and $\mathbf{d}$ is the nodal demand vector. Considering an error $\Delta \mathbf{d}$ in predicted demands $\mathbf{d}^{\mathbf{p}}$ that produces an error $\Delta \mathbf{h}$ in predicted heads $\mathbf{h}^{\mathbf{p}}$, the linear approximation is computed as

$$
\begin{gathered}
-\mathrm{BCB}^{T}\left(\mathbf{h}^{\mathbf{p}}+\Delta \mathbf{h}\right)=\mathbf{d}^{\mathbf{p}}+\Delta \mathbf{d} \\
-\mathrm{BCB}^{T} \Delta \mathbf{h}=\Delta \mathbf{d}
\end{gathered}
$$

Defining $\mathbf{A}=-\mathbf{B C B}^{T}$,

$$
\Delta \mathbf{h}=\mathbf{A}^{-1} \Delta \mathbf{d}
$$

Matrix $\mathbf{A}^{-1}$ in Eq. 8 is the complete sensitivity matrix relating changes in demands to changes in heads. If only a few head measurements are available, the new system of equations is redefined in Eq. 9.

$$
\mathbf{A}_{\mathrm{mh}} \Delta \mathbf{d}=\Delta \mathbf{h}_{\mathrm{mh}}
$$

Matrix $\mathbf{A}_{\mathbf{m h}}$ and vector $\Delta \mathbf{h}_{\mathbf{m h}}$ are extracted from matrix $\mathbf{A}^{-1}$ and vector $\Delta \mathbf{h}$, respectively. Subscript $\mathbf{m b}_{\mathbf{m}}$ refers to measured heads. Matrix $\mathbf{A}_{\mathbf{m h}}$ is the sensitivity matrix relating changes in demands to changes in measured heads.

The same process is applied to the flow equation defined in Eq. 10.

$$
\mathbf{q}=-\mathbf{C B}^{T} \mathbf{h}
$$

where $\mathbf{q}$ is the vector of flows. Considering an error $\Delta \mathbf{q}$ in predicted flows $\mathbf{q}^{\mathbf{p}}$ that produces an error $\Delta \mathbf{h}$ in predicted heads $\mathbf{h}^{\mathbf{p}}$, the perturbation equation is computed as 


$$
\begin{gathered}
\mathbf{q}^{\mathbf{p}}+\Delta \mathbf{q}=-\mathbf{C B}^{T}\left(\mathbf{h}^{\mathbf{p}}+\Delta \mathbf{h}\right) \\
\Delta \mathbf{q}=-\mathbf{C B}^{T} \Delta \mathbf{h}
\end{gathered}
$$

Replacing $\Delta \mathbf{h}$ with the one defined in Eq. 8:

$$
\Delta \mathbf{q}=-\mathbf{C B}^{T} \mathbf{A}^{-1} \Delta \mathbf{d}
$$

Matrix $\mathbf{A}_{\mathbf{m q}}$ and vector $\Delta \mathbf{q}_{\mathbf{m q}}$ are extracted from the result of $-\mathbf{C B}^{T} \mathbf{A}^{-1}$ and vector $\Delta \mathbf{q}$, respectively. Subscript $\mathbf{m q}$ refers to measured flows. Matrix $\mathbf{A}_{\mathbf{m q}}$ is the sensitivity matrix relating changes in demands to changes in measured flows.

$$
\mathbf{A}_{\text {mq }} \Delta \mathrm{d}=\Delta \mathbf{q}_{\mathbf{m q}}
$$

Both initial sensitivity matrices are calculated using demand allocation based on quarterly billing, as described in Eq. 14.

$$
\mathrm{d}_{i}(\mathrm{t})=\mathrm{bd}_{i} \cdot \mathrm{q}_{n}(\mathrm{t})
$$

where $\mathrm{d}_{i}(\mathrm{t})$ is the demand of node $\mathbf{i}$ at sample $\mathbf{t}$; $\mathbf{b d}_{i}$ is the base demand of node $\mathbf{i}$ computed from quarterly billing and fulfilling $\sum \mathbf{b d}_{i}=1$; and $\mathbf{q}_{n}(\mathrm{t})$ is the total consumed demand measured at the network inputs at sample $t$.

\section{Nodal demand calibration}

This work presents an implicit calibration methodology that uses the SVD for solving the inverse problem. The nodal demand vector $\mathbf{d}$ is computed through the minimisation of the objective function:

$$
\operatorname{min.} \quad J(\mathbf{d})=\sum_{i=1}^{n_{\mathrm{h}}}\left(\mathbf{w}_{i}^{h}\right)^{2}\left[\mathbf{h}_{i}^{m}-\mathbf{h}_{i}^{p}(\mathbf{d})\right]^{2}+\sum_{j=1}^{n_{\mathrm{f}}}\left(\mathbf{w}_{j}^{q}\right)^{2}\left[\mathbf{q}_{j}^{m}-\mathbf{q}_{j}^{p}(\mathbf{d})\right]^{2}
$$

subject to $\mathrm{G}\left(\mathbf{h}^{\mathrm{p}}, \mathbf{d}, \mathbf{r}\right)=0$

Where $\mathbf{J}$ is the objective function; $\mathrm{h}_{i}^{m}$ and $\mathrm{h}_{i}^{p}$ are the measured and predicted heads at node $\mathbf{i}$, respectively; $\mathbf{q}_{j}^{n}$ and $\mathbf{q}_{j}^{p}$ are the measured and predicted flows at pipe $\mathbf{j}$, respectively; $\mathbf{w}^{\mathbf{h}}$ and $\mathbf{w}^{\mathbf{q}}$ are the weighting factors applied to the different terms to ensure that they are of similar magnitude and unit; $\mathrm{n}_{h}$ and $\mathrm{n}_{f}$ are the number of measured nodal heads and pipe flows, respectively; $\mathbf{r}$ is the vector of pipes' roughness; and $\mathrm{G}$ is the system of non-linear equations describing the hydraulic steady state of flows and pressures in a WDN, including mass continuity and energy conservation equations, already described in Eq. 6 and 10. The formulation of the generalised inverse problem in Eq. 1 for WDN is:

$$
\mathrm{S} \cdot \Delta \mathrm{d}=\mathrm{e}
$$

where $\mathbf{S}$ is a concatenation of the sensitivity matrices $\mathbf{A}_{\mathbf{m h}}$ and $\mathbf{A}_{\mathbf{m q}}$; and $\mathbf{e}$ is a combination of prediction errors in measured heads and flows.

$$
\left|\begin{array}{c}
\mathbf{A}_{\mathbf{m h}} \\
\mathbf{A}_{\mathbf{m q}}
\end{array}\right| \Delta \mathbf{d}=\left|\begin{array}{c}
\Delta \mathbf{h}_{\mathbf{m h}} \\
\Delta \mathbf{q}_{\mathbf{m q}}
\end{array}\right|
$$


A constraint is added defining that the sum of consumptions can not vary:

$$
1 \Delta d=0
$$

where 1 is a row vector of ones with length equal to the number of demands. Weights $\mathbf{W}$ are added in order to unify units.

$$
\mathrm{W}\left|\begin{array}{c}
\mathbf{A}_{\mathbf{m h}} \\
\mathbf{A}_{\mathbf{m q}} \\
\mathbf{1}
\end{array}\right| \Delta \mathbf{d}=\mathbf{W}\left|\begin{array}{c}
\Delta \mathbf{h}_{\text {mh }} \\
\Delta \mathbf{q}_{\mathbf{m q}} \\
0
\end{array}\right|
$$

The SVD determines $\Delta \mathbf{d}$ for a problem that minimises $\|\Delta \mathbf{d}\|_{2}$ and $\|\mathbf{e}\|_{2}$. As the WDN is represented by a non-linear model, $\Delta \mathbf{d}$ is calculated iteratively, and used to correct the demand vector $\mathbf{d}$ :

$$
\mathbf{d}_{r+1}=\mathbf{d}_{r}+\rho \Delta \mathbf{d}_{r}
$$

At each iteration, the sensitivity matrices are computed using the resulting estimated demands of the previous iteration.

\section{Parameterization}

Calibration of nodal demands in a real life network is an underdetermined problem due to the low number of available sensors. The installation of extra sensors depends on the budget, which is generally limited. However, even with a high number of sensors, the calibration of individual demands is unattainable. Consequently, the parameters of the model have to be reduced by grouping demands depending on their behaviour.

Application of the SVD

So far in this work, the SVD has been used to compute the inverse of the sensitivity matrix for solving the calibration problem. However, the matrices obtained from the decomposition have further applications. Wiggins (1972) stated that "we can think of the eigenvectors $\mathbf{v}_{i}$ where $\mathbf{i}=1 \ldots . \mathrm{n}$ as a new parameterization of the model. These vectors represent a set of $\mathrm{n}$ linear combinations of the old parameters that are fixed by the observations". Matrix $\mathbf{V}$ is reduced into $\mathbf{V}_{\mathbf{r}}$, which is formed by the first $\mathrm{n}_{p}$ vectors $\mathbf{v}_{i}$, where $\mathrm{n}_{p}$ is the number of non-zero singular values of the sensitivity matrix. The new parameterization is obtained by defining a new parameter correction (Eq. 21).

$$
\Delta \mathrm{x}^{*}=\mathrm{V}_{\mathbf{r}}^{T} \Delta \mathrm{x}
$$

In WDN very low singular values appear, thus $\mathrm{n}_{p}$ is defined in a way that all values below the $\mathrm{n}_{p}$ highest singular values are neglected. Furthermore, the consideration of very low singular values leads to the increment of uncertainty (Aster et al. 2005). The main drawback of this approach is the loss of the physical meaning of the calibrated parameters as they would be generated by a different combination of the old parameters at each iteration. The sensors' data will be fitted, but the calibrated parameters will not have a direct relation with the WDN. 


\section{Introducing demand patterns}

The pressure and flow sensors used to monitor the network are not the unique source of information available. The water company manages information about the quarterly billing and type of consumer of each contract within the network. The first gives information about the average consumption of each contract, i.e. the weight (base demand) of this consumption over the whole network; while the second allows to discern among different temporal behaviours (patterns). These patterns are assumed to have daily periodicity. Consequently, demand of each node at each sample can be calculated as shown in Eq. 22 .

$$
\mathrm{d}_{i}(\mathrm{t})=\mathrm{bd}_{i} \cdot \mathrm{p}_{a \rightarrow i}(\mathrm{t}) \cdot \mathrm{q}_{n}(\mathrm{t})
$$

where $\mathbf{p}_{a \rightarrow i}(t)$ is the value of the pattern $\mathbf{p}_{a}$ associated to node $\mathbf{i}$ at sample $\mathbf{t}$. Considering nodal demands as defined in Eq. 22, the demand vector $\mathbf{d}=\left[\begin{array}{llll}\mathbf{d}_{1} & \mathrm{~d}_{2} & \ldots & \mathrm{d}_{n}\end{array}\right]^{T}$ can be expressed as seen in Eq. 23.

$$
\mathrm{d}(\mathrm{t})=\mathrm{BDM} \cdot \mathrm{TPM} \cdot \mathrm{p}(\mathrm{t}) \cdot \mathrm{q}_{n}(\mathrm{t})
$$

where $\mathbf{B D M}$ is the Base Demand Matrix, a diagonal $\mathrm{n} \times \mathrm{n}$ matrix containing the base demand values of each node; TPM is the Type of Pattern Matrix, an $\mathbf{n} \times \mathbf{n}_{p}$ matrix associating each initial parameter (nodal demand) with a new parameter (demand pattern); and $\mathbf{p}(\mathrm{t})$ is a vector containing $\mathrm{n}_{p}$ patterns at sample $\mathrm{t}$.

Assuming that BDM and TPM matrices are known and fixed during time, variations in demands $\Delta \mathbf{d}(\mathrm{t})$ will be caused by variations in patterns $\Delta \mathrm{p}(\mathrm{t})$, as defined in Eq. 24.

$$
\Delta \mathrm{d}(\mathrm{t})=\mathrm{BDM} \cdot \mathrm{TPM} \cdot \Delta \mathrm{p}(\mathrm{t}) \cdot \mathrm{q}_{n}(\mathrm{t})
$$

Consequently, Eq. 19 becomes:

$$
\mathbf{W}\left|\begin{array}{c}
\mathbf{A}_{\mathbf{m h}}(\mathrm{t}) \\
\mathbf{A}_{\mathbf{m q}}(\mathrm{t}) \\
\mathbf{1}
\end{array}\right| \cdot \mathrm{BDM} \cdot \mathbf{T P M} \cdot \Delta \mathbf{p}(\mathrm{t}) \cdot \mathbf{q}_{n}(\mathrm{t})=\mathbf{W}\left|\begin{array}{c}
\Delta \mathbf{h}_{\mathbf{m h}}(\mathrm{t}) \\
\Delta \mathbf{q}_{\mathbf{m q}}(\mathrm{t}) \\
0
\end{array}\right|
$$

The SVD determines $\Delta \mathbf{p}$ for a problem that minimises both $\|\Delta \mathbf{p}\|_{2}$ and $\|\mathbf{e}\|_{2}$. Solving iteratively Eq. 25 leads to a set of patterns values for sample t.

The SVD for distribution of patterns

The definition of patterns is a key factor for the viability of the methodology. Assigning patterns to each demand depending on their type of contract is a logical way forward. However, the mixing of contracts in cities provokes changes in sensors' measurements that can not be assigned to a unique pattern (Sanz and Pérez 2014a). From the calibration point of view, it would be desirable to have geographical patterns, so the sensors' changes could depend mostly on a single parameter. This geographical distribution can be obtained by analysing the $\mathbf{V}$ matrix from the SVD as explained on the first part of this section.

Matrix $\mathbf{V}$ gives information about the parameter resolution $\mathbf{R}=\mathbf{V V}^{T}$, which describes how the generalised inverse solution smears out the original model $\mathbf{x}$ 


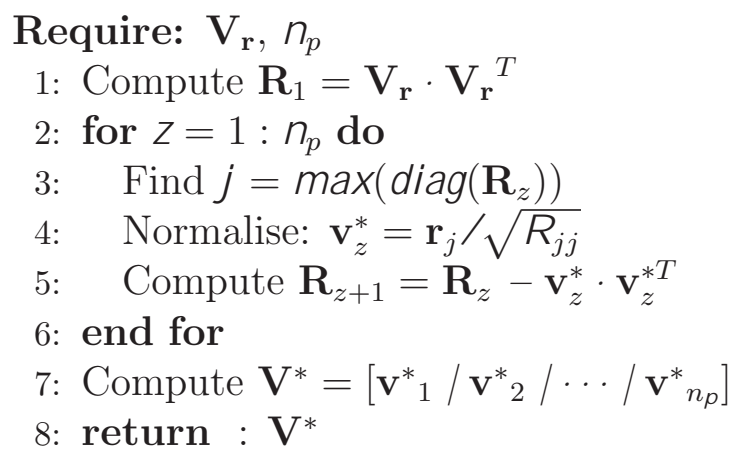

\section{Figure 1. Pseudo-code of the delta vector generation for parameteriza- tion}

of Eq.1 into a recovered model $\hat{\mathbf{x}}$ (Aster et al. 2005). A perfect resolution is represented by the identity matrix, indicating that each parameter is perfectly resolved. Unfortunately, when only $\mathrm{n}_{p}$ parameters corresponding to the highest $\mathrm{n}_{p}$ singular values are considered, the resolution matrix computed with $\mathbf{V}_{\mathbf{r}}$ is not the identity matrix. Compact resolution appears and parameters with similar sensitivities can be identified. This identification is performed by means of the "delta vector generation" process (Wiggins 1972), which is used to define the TPM matrix with the membership of each individual demand to each demand pattern. The resulting parameterization is used to calibrate groups of demands.

The estimated nodal demands will not behave exactly as the real ones, but it is expected to get group behaviours (demands and pressures) similar to the real values. These similarities will be used as indicators of the calibration's performance.

Pseudo-code shown in Fig. 1 summarises the delta vector generation process, where the $\mathbf{n}_{p}$ vectors with the highest resolving power in the resolution matrix are obtained and normalised iteratively to generate the delta vectors.

The $\mathbf{v}^{*}$ delta vectors are then used to generate the TPM, associating each initial parameter (consumption) to a new parameter (pattern) that produces the best resolution if $\mathrm{n}_{p}$ patterns are considered. Three approaches are proposed: binary parameterization, positive hybrid parameterization or free hybrid parameterization. The first approach assigns a single demand pattern to each nodal demand (Sanz and Pérez 2014b); the second approach assigns a combination of demand patterns to each nodal demand with positive weights; the latter approach considers a combination of patterns that can include negative weights.

Pseudo-code shown in Fig. 2 presents the generation of the accumulated $\overline{\mathbf{T P M}}$ matrix with positive hybrid parameterization using the sensitivity matrices from $\mathrm{n}_{t}$ samples. For the free hybrid parameterization, line 4 is eliminated. The normalisation of the rows in $\mathbf{V}^{*}$ is done so that the weights can be interpreted as memberships of each nodal demand to each pattern.

The solution tends to generate geographical patterns, as the topological in- 


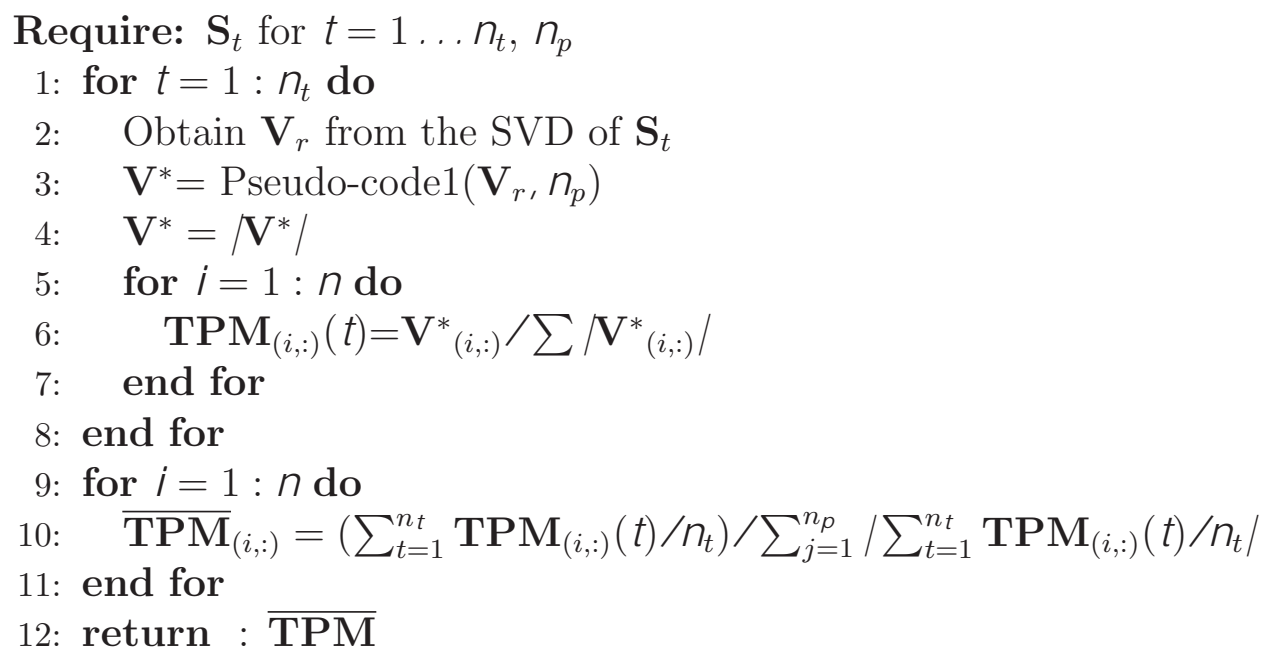

Figure 2. Pseudo-code of the accumulated TPM generation with positive hybrid parameterization

formation (incidence matrix B) is included in the sensitivity matrix, as seen in Eq. 7. The samples used in this process belong to different hours, and the mean value for each weight is calculated. Due to the static topology of the network the sensitivity matrix is not expected to have significant variations, so the mean value is representative for all the used hours.

The calibration methodology requires some inner sensors to be distributed through the sampling design. In case the network already has the sensors installed, the $\mathbf{S}$ matrix introduced in pseudo-code shown in Fig. 2 would be a reduced sensitivity matrix $\mathbf{S}_{\mathbf{r}}$ where only the rows related to the available sensors would be considered.

\section{Sampling Design}

The information extracted from the network depends highly on the location of the sensors. Each added sensor represents a new equation in the system. In order to have a determined system of equations, the number of measurements (sensors) has to be equal to the number of parameters, guaranteeing the system identifiability in the linear approximation. The sampling design is performed after the distribution of patterns, selecting the $\mathbf{n}_{p}$ best sensors. The process for locating the sensors uses matrix $\mathbf{U}$ in the same way as the parameterization process uses matrix $\mathbf{V}$.

The information density matrix $\mathbf{I}_{\mathbf{d}}=\mathbf{U U}^{T}$ describes how the generalised inverse solution smears out the original data $\mathbf{y}^{\mathbf{m}}$ in Eq. 1 into a predicted data $\mathbf{y}^{\mathbf{p}}$ (Aster et al. 2005). Matrix $\mathbf{U}$ is computed from the sensitivity matrix $\mathbf{S}^{*}$ (Eq. 26), which is obtained considering the new parameterization.

$$
\mathrm{S}^{*}=\mathbf{S} \cdot \overline{\mathbf{T P M}}
$$




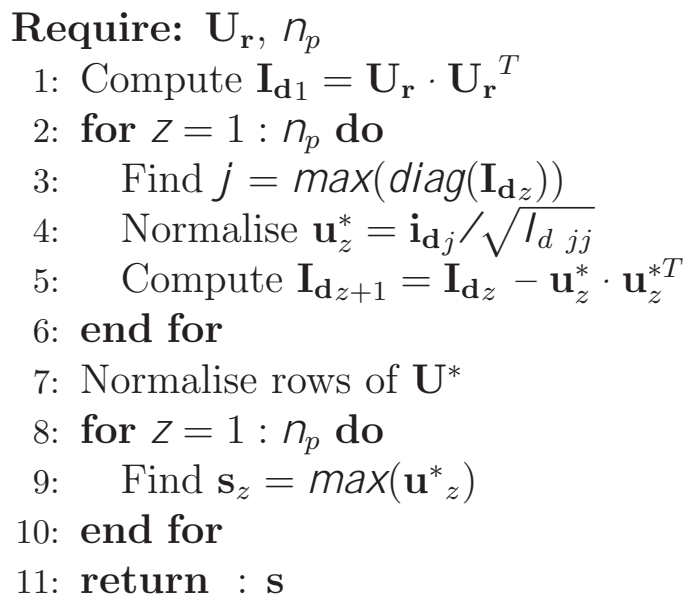

Figure 3. Pseudo-code of the delta vector generation for sensors selection

where $\mathbf{S}^{*}$ is a $\mathrm{m \times n} \mathrm{n}_{p}$ matrix describing the changes in potential measurements due to changes in demand patterns.

Then, the delta vector generation process is applied to matrix $\mathbf{U}$ in order to obtain the delta vectors that give a measure of the sensitivity of each potential sensor to each demand pattern. The rows of the delta vectors are normalised, so the sensors with high sensitivity to a pattern and low sensitivity to the others are selected. Pseudo-code shown in Fig. 3 summarises this process.

The output of the pseudo-code is a sensor vector $\mathbf{s}$ with length $\mathrm{n}_{p}$ containing the most sensitive sensor for each demand pattern. The pseudo-code can be applied to different time samples, selecting at the end of the process the most repeated sensors. In both parameterization and sampling design approaches the delta vectors are calculated sequentially so the effect of selecting a pattern or sensor is considered when selecting the next one.

\section{Reducing the uncertainty}

The stochastic nature of demands and the noise in measurements cause the calibrated patterns to have high uncertainty. Two actions are proposed to reduce it.

\section{Oversampling}

The reduction of the sensors' noise can be achieved by filtering several measurements along a period of time, reducing the standard deviation of the averaged measurement (Eq. 27) while increasing its resolution (Pandya and Gupta 2014).

$$
\sigma_{\text {filtered }}=\vee_{\overline{\mathrm{n}_{k}}}^{1} \cdot \sigma_{\text {measurement }}
$$

where $\mathrm{n}_{k}$ is the number of measurements done along the same time period. 


\section{Including extra data}

The inherent noise in demands that affects the calibrated values can be reduced if data from multiple samples with the same boundary conditions and same expected demand patterns behaviours are used. The system becomes overdetermined, and the solution minimises the error of all samples simultaneously. The system presented in Eq. 25 is extended including the extra data samples, as shown in Eq. 28.

$$
\mathbf{W}\left|\begin{array}{c}
\mathbf{A}_{\mathbf{m h}}\left(\mathrm{k}_{1}\right) \cdot \mathrm{q}_{n}\left(\mathrm{k}_{1}\right) \\
\vdots \\
\mathbf{A}_{\mathbf{m h}}\left(\mathrm{k}_{n}\right) \cdot \mathrm{q}_{n}\left(\mathrm{k}_{n}\right) \\
\mathbf{A}_{\mathbf{m q}}\left(\mathrm{k}_{1}\right) \cdot \mathrm{q}_{n}\left(\mathrm{k}_{1}\right) \\
\vdots \\
\mathbf{A}_{\mathbf{m q}}\left(\mathrm{k}_{n}\right) \cdot \mathrm{q}_{n}\left(\mathrm{k}_{n}\right) \\
\mathbf{1}
\end{array}\right| \cdot \mathbf{B D M} \cdot \overline{\mathbf{T P M}} \cdot \Delta \mathbf{p}\left(\mathrm{t} \rightarrow \mathrm{k}_{1} \ldots \mathrm{k}_{n}\right)=\mathbf{W}\left|\begin{array}{c}
\Delta \mathbf{h}_{\mathbf{m h}}\left(\mathrm{k}_{1}\right) \\
\vdots \\
\Delta \mathbf{h}_{\mathbf{m h}}\left(\mathrm{k}_{n}\right) \\
\Delta \mathbf{q}_{\mathbf{m q}}\left(\mathrm{k}_{1}\right) \\
\vdots \\
\Delta \mathbf{q}_{\mathbf{m q}}\left(\mathrm{k}_{n}\right) \\
0
\end{array}\right|
$$

The inclusion of the extra samples does not increase the rank of the sensitivity matrix. However, the condition number decreases (Aster et al. 2005), reducing the propagation of noise from measurements to demand patterns (Eq. 5).

\section{CASE STUDY}

The calibration methodology is applied to a real network with synthetic data. The network is a district metered area (DMA) situated in the Barcelona neighbourhood of Nova Icaria. It is composed of 3455 pipes and 3377 junctions, as depicted in Fig. 4. Water is supplied to the network through two pressure reduction valves, highlighted in Fig. 4 with a triangle and a circle. Pressure and flow are monitored at both water inlets with a sample time of 10 minutes. The resolution of the sensors is $0.01 \mathrm{l} / \mathrm{s}$ for the flow sensors, and $0.01 \mathrm{mwc}$ (meters of water column) for both the inlet and inner pressure sensors.

\section{Synthetic data generation}

Synthetic nodal demands have been generated with a random normal noise $\mathbf{N}\left(0,0.1 \mathbf{d}_{i}(\mathbf{t})\right)$ applied to each demand at each sample, where $\mathbf{d}_{i}(\mathbf{t})$ is the consumption of node $\mathbf{i}$ at sample $\mathbf{t}$ without noise. Ten patterns of behaviour have been defined, representing different types of contracts: industrial, restaurant, commercial, etc. All patterns and consequently all nodal demands have different behaviours during weekdays and weekends. The average consumption of each node has been calculated with the mean of the three months' synthetic demands. A random normal noise $\mathbf{N}(0,0.01 \mathrm{mwc})$ is added to the simulated inner sensors' measurements.

\section{Calibration assumptions}

The output of the calibration methodology is the set of patterns values that minimises the errors on the measured pressures for a specific hour. Extra data can be used to calibrate a single pattern value if the boundary conditions are maintained. The current work assumes that weekdays' behaviours are similar. Consequently, sensors' values from the last five weekdays are used to calculate $\|\mathbf{e}\|_{2}$ 


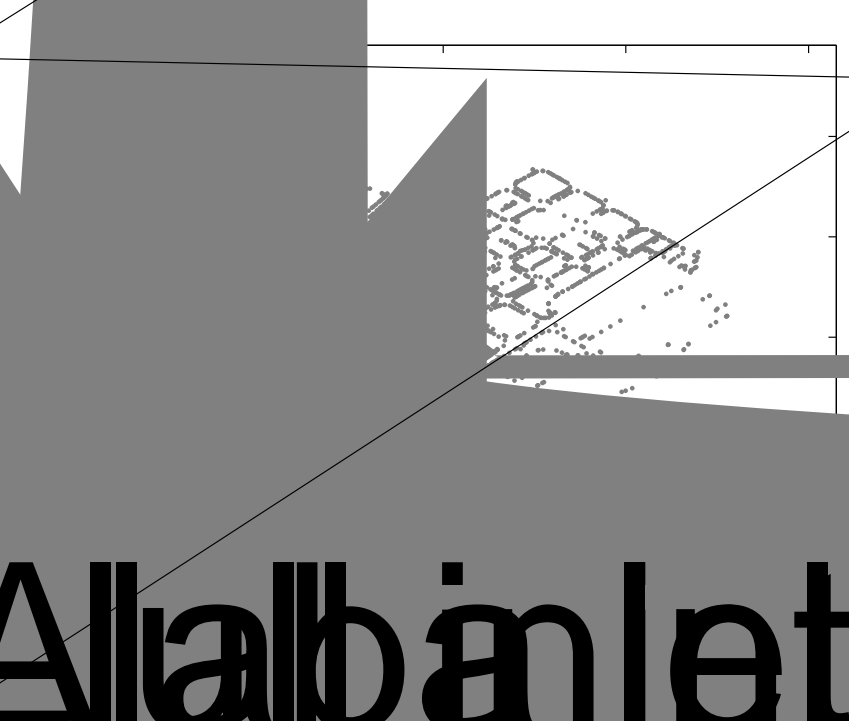

Figure 4. Nova Icaria DMA EPANET model with highlighted inputs

for a specific hour. The corresponding calibrated patterns values minimise the error at that hour. A more detailed calibration can be performed by considering weekdays separately, using extra data from the same day in previous weeks.

The real-time calibration modifies demand patterns continuously to adapt to seasonal changes. The use of historical data is restricted by these changes. As mentioned before, this work considers data from the current day and the four previous weekdays. Weekends would be calibrated separately.

\section{RESULTS}

\section{Parameterization}

The first step towards calibration consists in defining the parameters to be calibrated. The number of demand patterns that are going to be calibrated depends on the size of the network and the budget, as the number of sensors to be installed has to be the same or higher than the number of patterns. In the current study five patterns are considered following the company assessment. The three types of patterns' weights explained in previous sections (binary, positive and free) have been tested. The calibration results for each of the distributions and a comparison between them are presented.

Initially, the sensitivity matrix is analysed. Similar behaviours are observed in all samples due to the topological information included in the sensitivity matrix. The resulting distributions are depicted in Fig. 5. The membership of each nodal demand to each pattern is marked in black/white for the binary weights; greyscale for the positive weights; and cold/hot scale for the free weights. It can be seen that a geographical distribution of patterns appears. This means that consumptions' changes in a zone affect similarly to all pressures. 

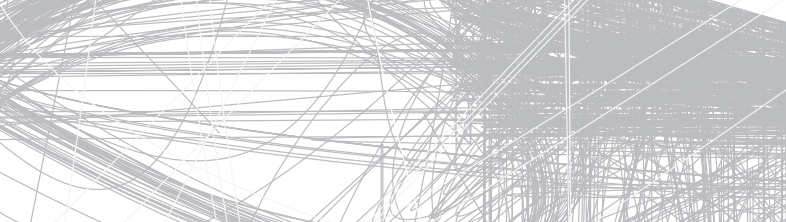

Figure 5. Distributions of patterns considering binary weights, positive weights and free weights

Table 1. Percentage of consumption for each distribution

\begin{tabular}{cccc}
\hline \hline Type of weights & Binary & Positive & Free \\
\hline Pattern 1 & $12.5 \%$ & $23 \%$ & $22 \%$ \\
Pattern 2 & $8.5 \%$ & $13.7 \%$ & $12.8 \%$ \\
Pattern 3 & $22.7 \%$ & $18.2 \%$ & $14.4 \%$ \\
Pattern 4 & $41.8 \%$ & $29.5 \%$ & $33.4 \%$ \\
Pattern 5 & $14.5 \%$ & $15.6 \%$ & $17.4 \%$ \\
\hline \hline
\end{tabular}

Table 1 summarises the percentage of consumption during three months of each pattern calculated from the nodal quarterly billing and the membership of each demand to each pattern for the three types of weights.

\section{Sampling Design}

The sensor distribution is performed after the definition of patterns. Results obtained for each of the distributions have been the same. This is due to the similarity between the three parameterizations. Fig. 6 depicts the locations of the selected pressure sensors with diamond shapes, each one situated inside a different pattern effect area.

\section{Demand Calibration}

The demand pattern calibration methodology is applied to calibrate a weekday pattern of 24 hours, with a unique pattern value per hour. Oversampling is considered by filtering six samples inside each hour, taken every ten minutes. Additionally, data from the five weekdays are included as described in Eq. 28, assuming similar demand behaviour during working days. 


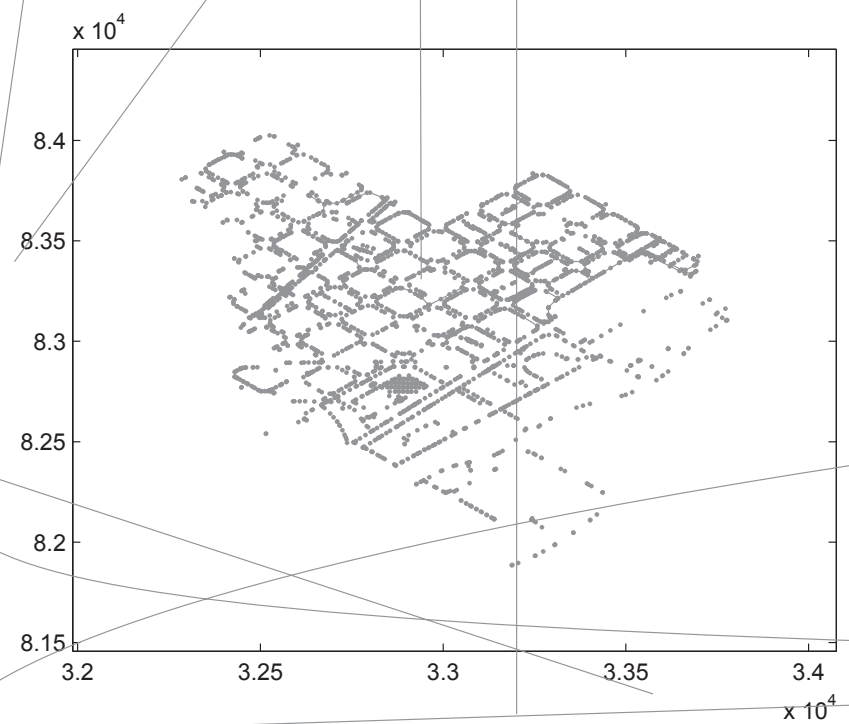

Figure 6. Selected sensors in Nova Icaria DMA using the SVD

Table 2. Mean squared errors in cumulative demands, pressures and flows for each pattern distribution

\begin{tabular}{cccc}
\hline \hline Type of weights & Binary & Positive & Free \\
\hline Cumulative demand & 1.76 & $\mathbf{0 . 1 6}$ & 9.93 \\
Pressures & 0.95 & $\mathbf{0 . 3 7}$ & 0.41 \\
Flows & 0.05 & $\mathbf{0 . 0 3}$ & 0.06 \\
\hline \hline
\end{tabular}

First, results from the three presented parameterization approaches are compared. Fig. 7 depicts the calibrated patterns for each parameterization. It can be seen that patterns' shapes are similar in all cases, but with different scale factors. Low uncertainty is obtained due to oversampling and addition of extra data.

A second indicator to validate results is shown in Fig. 8, where the cumulative demands obtained from billing (circles) and from estimated patterns (asterisks) are compared. The positive weights approach gives the best result in terms of this indicator, while the free weights approach generates the highest error.

Finally, the prediction errors on pressures and flows are compared. The mean squared errors (mse) of the cumulative demand (first row), predicted pressures (second row) and predicted flows (third row) are summarised in Table 2. The cumulative demand errors are computed from the numerical values in Fig. 8. The positive weights pattern distribution generates the lowest prediction error in all indicators.

\section{CONCLUSIONS}

This work presents a complete process for calibrating demands in a water distribution system. Reducing the number of parameters to be calibrated is the 

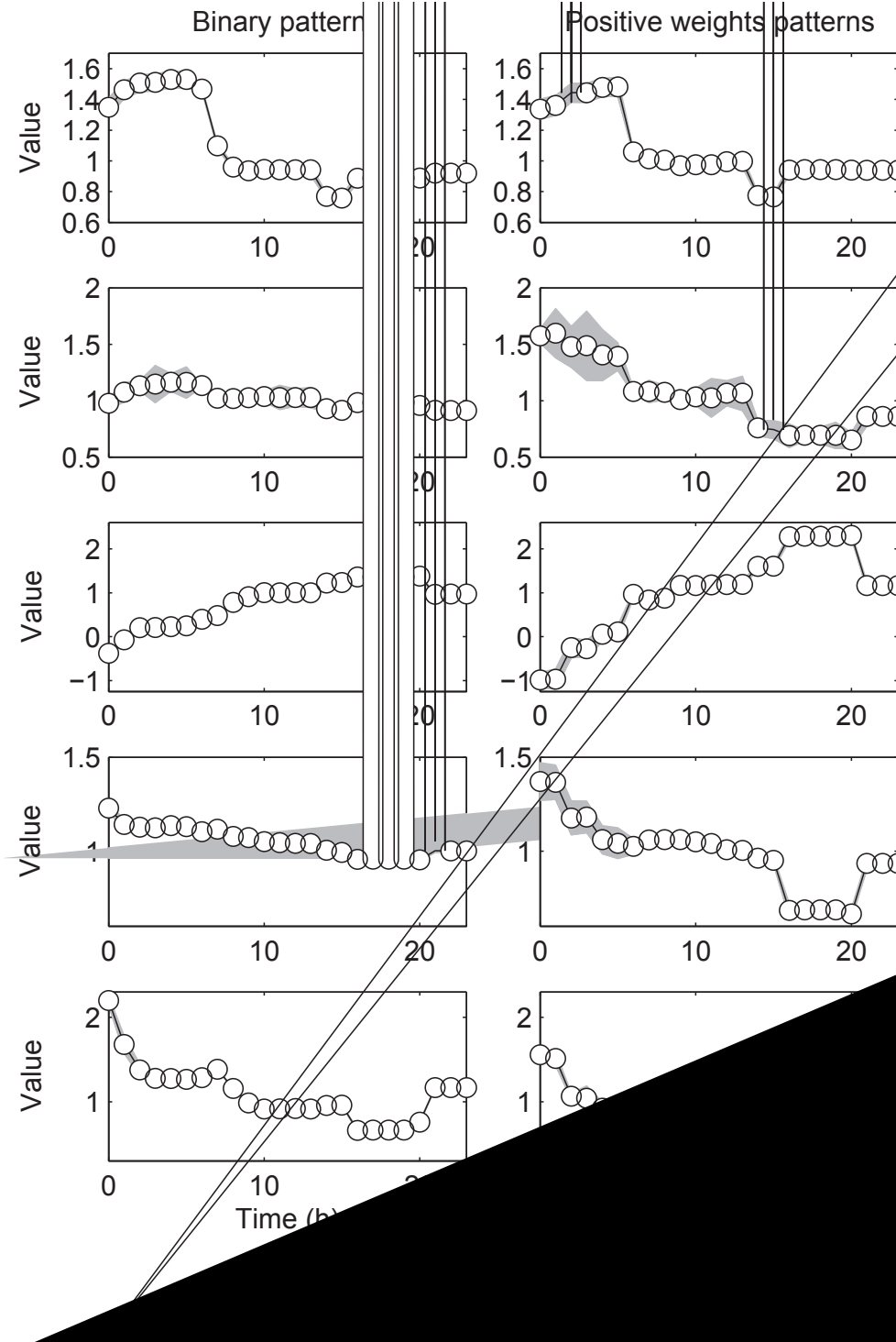

Figure 7. Calibrated patterns and uncertainty with three different pattern distributions

key factor to make the system identifiable. The introduction of demand patterns allows to redefine individual demands by combining different demand behaviours. A good selection of both patterns and sensors is required.

The SVD is a powerful tool for solving the calibration problem. Furthermore, it allows to analyse deeply the system behaviour, leading to a good understanding of the parameterization and sampling design problems.

First, three approaches for defining the membership of each individual demand (initial unknown parameter) to each demand pattern are presented. The difference between each approach consists in the type of weights considered: binary, positive and free. These weights are obtained by analysing the resolution matrix computed 

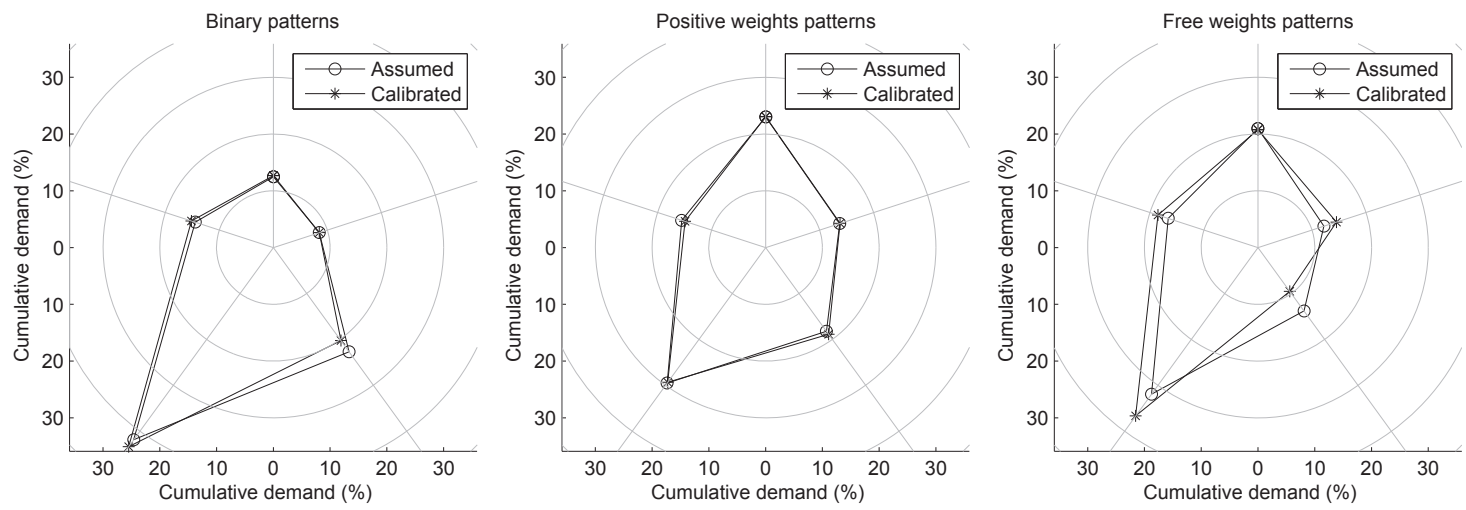

Figure 8. Percentage of assumed and predicted cumulative demand for each of the three pattern distributions

from the parameter space matrix generated through the SVD of the sensitivity matrix.

Once the new parameters are defined, a similar process is followed to select the sensors that have high sensitivity to a single pattern and low sensitivity to the rest. This selection is obtained from the analysis of the information density matrix, calculated from the data space matrix. Data from along the day have been used for the parameter definition and sensor selection processes. The sensitivity all over the day has been analysed showing unimportant variations.

Finally, the generalised inverse problem is solved iteratively by means of the SVD to compute hourly demand patterns. The FOSM model calculated with matrices from the SVD is used to propagate the uncertainty from sensors measurements to calibrated parameters. Oversampling and data accumulation help reduce the uncertainty from real instant demand values and sensors precision.

The proposed methodologies are tested on a real network with synthetic data. These data are based on real quarterly billing and ten synthetic demand patterns. Considering the test as a real application, five demand patterns are defined, and the five best sensors are selected. The calibration process is performed using the three types of weights explained. The evaluation of the results is done considering the same conditions as in a real case: cumulative percentage of demand for each pattern, error on predicted pressures and error on predicted flows. The positive weights pattern distribution generates the best results in terms of the evaluated indicators.

The geographical distribution of patterns is a potential tool for a future fault detection methodology. The calibration process is performed online, and the calibrated patterns are compared with historical values in order to detect unexpected changes that may be assigned to a fault and located in a specific area of the network. 


\section{NOTATION}

The following symbols are used in this paper:

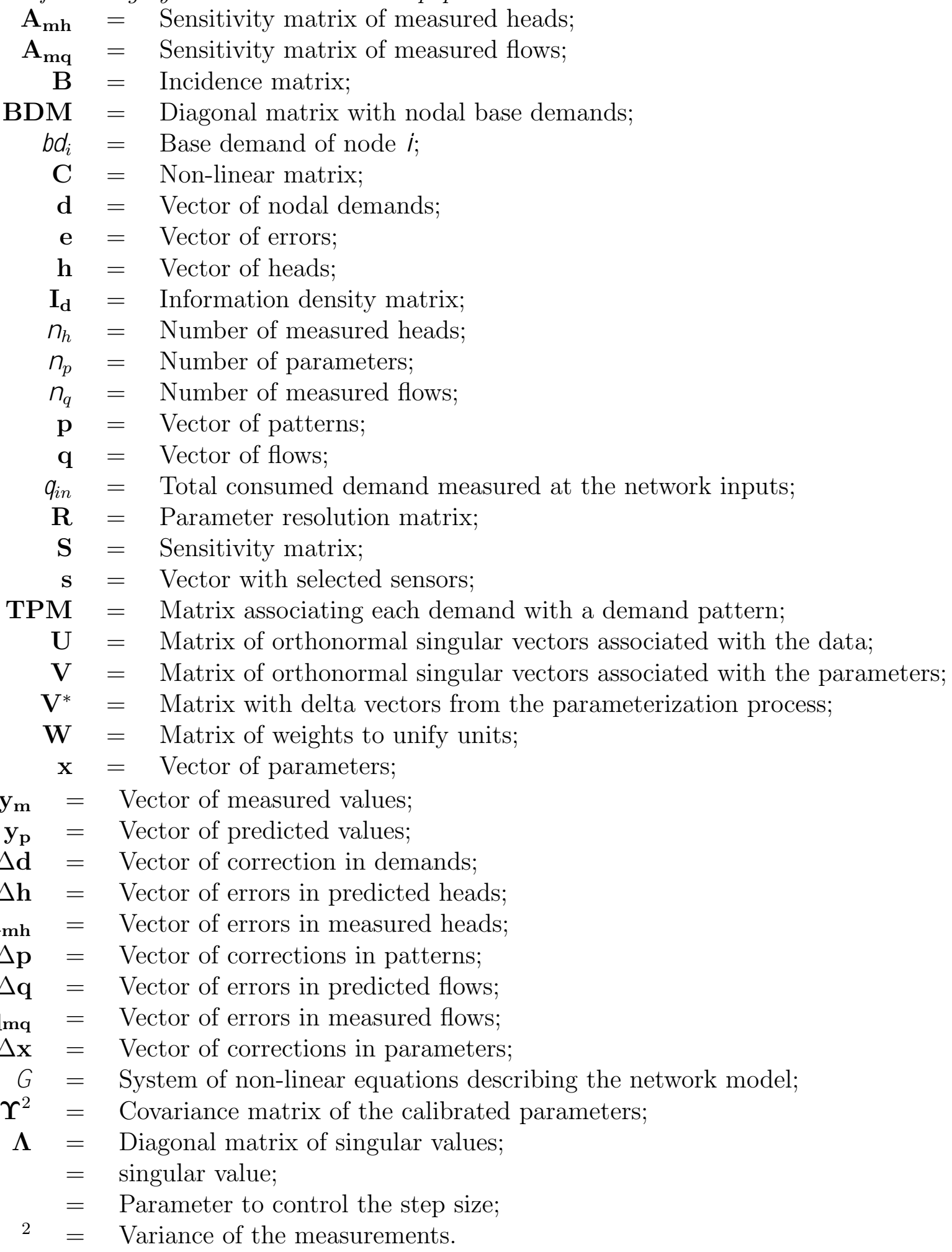

\section{ACKNOWLEDGEMENTS}

This work was supported in part by the project FP7-ICT-2012-318556 (EFFINET) 
of the European Commission and by the Polytechnic University of Catalonia. The model of the real network was provided by the Barcelona Water Company AGBAR. 


\section{REFERENCES}

Aster, R., Borchers, B., and Thurber, C. (2005). Parameter Estimation and Inverse Problems. Elsevier, New York.

Bard, Y. (1974). Nonlinear Parameter Estimation. Academic Press, San Diego, California.

Bryds, M. A. and Ulanicki, B. (1994). Operational Control of Water Systems: Structures, Algorithms and Applications. Prentice Hall International.

Cheng, W. and He, Z. (2011). "Calibration of Nodal Demand in Water Distribution Systems." Journal of Water Resources Planning and Management, 137(1), $31-40$.

Davidson, J. and Bouchart, F. (2006). "Adjusting Nodal Demands in SCADA Constrained Real-Time Water Distribution Network Models." Journal of Hydraulic Engineering, 132(1), 102-110.

Goulet, J.-A., Coutu, S., and Smith, I. F. (2013). "Model falsification diagnosis and sensor placement for leak detection in pressurized pipe networks." Advanced Engineering Informatics, 27(2), 261-269.

Hutton, C. J., Kapelan, Z., Vamvakeridou-Lyroudia, L., and Savić, D. A. (2014). "Dealing with Uncertainty in Water Distribution System Models: A Framework for Real-Time Modeling and Data Assimilation." Journal of Water Resources Planning and Management, 140(2), 169-183.

Kang, D. and Lansey, K. (2011). "Demand and Roughness Estimation in Water Distribution Systems." Journal of Water Resources Planning and Management, 137(1), 20-30.

Kapelan, Z., Savic, D., and Walters, G. (2005). "Optimal Sampling Design Methodologies for Water Distribution Model Calibration." Journal of Hydraulic Eng., 131(3), 190-200.

Menke, W. (1982). Geophysical Data Analysis: Discrete Inverse Theory. Academic Press.

Nicolini, M., Giacomello, C., and Deb, K. (2011). "Calibration and Optimal Leakage Management for a Real Water Distribution Network." Journal of Water Resources Planning and Management, 137(1), 134-142.

Pandya, P. and Gupta, V. (2014). "Enhancing Analog to Digital Converter Resolution Using Oversampling Technique." Blue Eyes Int. Eng. $\mathcal{B S}^{3}$ Sciences Publication Pvt. Ltd., 2(5).

Pérez, R., Nejjari, F., Puig, V., Quevedo, J., Sanz, G., Cugueró, M., and Peralta, A. (2011). "Study of the isolability of leaks in a network depending on calibration of demands." 11th International Conf. on Computing and Control for the Water Industry, Exeter, 455-460.

Sanz, G. and Pérez, R. (2014a). "Demand Pattern Calibration in Water Distribution Networks." Procedia Engineering, 70, 1495-1504.

Sanz, G. and Pérez, R. (2014b). "Parameterization and Sampling Design for Water Networks Demand Calibration using the Singular Value Decomposition: Application to a Real Network." 11th International Conference on HydroInformatics, New York. 
Savic, D., Kapelan, Z., and Jonkergouw, P. (2009). "Quo vadis water distribution model calibration?." Urban Water Journal, 6(1), 3-22.

Shamir, U. and Howard, C. (1977). "Engineering Analysis of Water-Distribution Systems." Journal of the American Water Works Association, 69(9), 510-514.

Sumer, D. and Lansey, K. (2009). "Effect of Uncertainty on Water Distribution System Model Design Decisions." J. of Water Resources Planning and Management, 135(1), 38-47.

Uhrhammer, R. (1980). "Analysis of Small Seismographic Station Networks." 8 Bulletin of the Seismological Society of America, 70(4), 1369-1379.

Walski, T. (1983). "Technique for Calibrating Network Models." Journal of Water Resources Planning and Management, 109(4), 360.

Walski, T., Chase, D., Savic, D., Grayman, W., Beckwith, S., and Koelle, E. (2003). Advanced Water Distribution Modeling and Management. Haestad Press.

Wasantha Lal, A. M. (1995). "Calibration of Riverbed Roughness." Journal of Hydraulic Engineering, 121(9), 664-671.

Wiggins, R. (1972). "The general linear inverse problem: Implication of surface waves and free oscillations for Earth structure." Reviews of Geophysics, 10(1), $251-285$.

Yeh, W. (1986). "Review of Parameter Identification Procedures in Groundwater Hydrology: The Inverse Problem." Water Resources Research, 22(2), 95-108. 